\title{
Human cytomegalovirus nuclear capsid motility is non-directed and independent of nuclear actin bundles
}

1 Felix Flomm ${ }^{\mathrm{a}^{*}}$, Eva Maria Borst ${ }^{\mathrm{b}^{*}}$, Thomas Günther ${ }^{\mathrm{a}}$, Rudolph Reimer ${ }^{\mathrm{a}}$, Laura de Vries ${ }^{\mathrm{a}}$,

2 Carola Schneidera, Adam Grundhoffa, Kay Grünewald ${ }^{c, d}$, Martin Messerle ${ }^{b, d}$, Jens Bern-

3 hard Bosse ${ }^{a} \#$

4 a Heinrich-Pette-Institut, Leibniz-Institut für Experimentelle Virologie, Hamburg, Germany

$5 \quad$ bepartment of Virology, Hannover Medical School, 30625 Hannover, Germany

$6{ }^{\mathrm{c} C e n t r e}$ of Structural Systems Biology (CSSB), Notkestr. 85, D-22607, Heinrich-Pette-Insti-

7 tute/ University of Hamburg, Hamburg, Germany

$8{ }^{d}$ Cluster of Excellence RESIST (EXC 2155), Hannover Medical School, Hannover, Germany.

9

$10 \quad{ }^{*} \mathrm{FF}$ and $\mathrm{EMB}$ contributed equally to this work

11 \#Address correspondence to Jens Bernhard Bosse, jens.bosse@leibniz-hpi.de.de

12

13

14 Running title: HCMV nuclear capsid motility

15

16 Keywords: Nucleus, F-actin, CMV, Single particle tracking, Microscopy 


\section{Abstract}

19 Herpesvirus genome replication, capsid assembly and packaging take place in the host cell

20 nucleus. Matured capsids leave the nucleus through a unique envelopment-de-envelopment

21 process at the nuclear membranes called nuclear egress. How assembled and DNA-contain-

22 ing herpesvirus capsids reach the sites of nuclear egress is however still controversially discussed, as host chromatin that marginalizes during infection might constitute a major barrier.

24 For alphaherpesviruses, previous work has suggested that nuclear capsids use active transport mediated by nuclear filamentous actin (F-actin). However, direct evidence for nuclear capsid motility on nuclear F-actin was missing. Our subsequent work did not detect nuclear F-actin associated with motile capsids, but instead found evidence for chromatin remodeling to facilitate passive capsid diffusion. A recent report described that human cytomegalovirus, a betaherpesvirus, induces nuclear F-actin and that the motor protein myosin $\mathrm{V}$ localizes to these structures. Direct evidence of capsid recruitment to these structures and

31 motility on them was however missing. In this study, we tested the functional role of HCMV-

32 induced, nuclear actin assemblies for capsid transport. We did not observe transport events 33 along nuclear F-actin. Instead, reproduction of nuclear F-actin was only possible using strong 34 overexpression of the fluorescent marker LifeAct-mCherry-NLS. Also, two alternative fluo35 rescent F-actin markers did not detect F-actin in HCMV-infected cells. Furthermore, single particle tracking of nuclear HCMV capsids showed no indication for active transport, which is in line with previous work on alphaherpesviruses.

39 Although human cytomegalovirus hardly causes disease in healthy individuals, it constitutes 40 a major hazard to immunocompromised risk groups. Human Cytomegalovirus infests nearly 
41 all organs and can cause severe disease such as pneumonitis, colitis, encephalitis and reti-

42 nitis, and can lead to serious impairments in neonates. Currently available treatments target

43 only two steps during the viral 'life cycle', which makes the occurrence of viral resistance a

44 major problem. To identify targets for pharmaceuticals, in-depth knowledge of the molecular mechanisms of the viral infection is paramount. Since the virus relies on the ability to release infectious particles from a host cell to infect another cell, its ability to translocate these particles within a cell is critical to complete the viral 'life cycle'. This work indicates that remodeling of cellular chromatin, rather than molecular motors, enables capsid access to the nuclear membrane. Understanding the mechanism of chromatin remodeling might help in designing effective inhibitors.

\section{Introduction}

$53 \mathrm{HCMV}$, like other herpesviruses, creates infectious particles using a series of complex mor54 phogenesis steps that lead to the fully assembled and infectious virion consisting of capsid, 55 tegument, and envelope. These layers are subsequently added while the forming particles 56 pass through several host-cell organelles $(1,2)$. Morphogenesis starts in the nucleus, where 57 DNA replication, the formation of nucleocapsids, as well as the packaging of viral genomes 58 into the latter takes place. Next, herpesvirus capsids must reach the nuclear envelope for 59 primary envelopment and egress (3). How nuclear capsids cross the nucleoplasm is contro60 versially discussed in the field. Earlier work suggested that herpes simplex virus type 1 (HSV-

61 1) capsids exhibit directed nuclear motility as determined by single particle tracking. The ob62 served directed motility could be antagonized by a putative myosin inhibitor as well as the 63 actin-depolymerizing drug Latrunculin A, suggesting that capsids might use myosins to move 64 actively on nuclear F-actin (4). One year later a study was able to detect F-actin in fixed rodent 
neuronal cells infected with pseudorabies virus (PRV), using both fluorescence microscopy as well as serial-block-face scanning electron microscopy (SBFSEM) (5). However, we recently did not detect any nuclear F-actin in fibroblasts infected with the alphaherpesviruses HSV-1, PRV, the betaherpesvirus murine cytomegalovirus (MCMV) or the gammaherpesvirus murine herpesvirus-68 (MHV-68), while nuclear capsid motility in these cells was detectable (6)

Moreover, we found that Latrunculin A was able to induce aberrant actin assemblies that seemed to unspecifically bind viral capsids and block their movement $(6,7)$. Since direct evidence of capsid motility along nuclear actin filaments was missing, and advances in camera technology now allow much more precise measurements, we re-evaluated previous findings. Using a custom microscope design, we acquired several thousand alphaherpesvirus nuclear capsid tracks. Analysis of these tracks showed no indication of bulk directed motility of nuclear herpesvirus capsids. Instead, we found that infection-induced chromatin remodeling allowed capsids to cross the nucleoplasm by diffusion to reach the nuclear envelope.

These findings are supported by two recent reports in which the authors were able to resolve interchromatin channels that bridge through the marginalized chromatin in HSV-1 infected cells to supposed egress sites at the nuclear envelope (8). Computational modeling using our experimentally determined diffusion coefficients indicates that these channels allow herpesvirus capsids to reach the nuclear envelope by diffusion with(9).

In discordance with these findings, a recent report showed large nuclear actin filaments in human foreskin fibroblast (HFF) cells stably expressing LifeAct-GFP-NLS and infected with HCMV (10). In addition, the authors found that prolonged incubation of infected cells with very high concentrations of the F-actin-depolymerizing drug Latrunculin A led to a defect in infectious virus production as well as their translocation to the cytoplasm, and suggested that these filaments are involved in movement of capsids to the nuclear periphery for nuclear egress. 
Moreover, myosin Va was implicated in nuclear egress, as the authors found a colocalization

91 of myosin Va with the major capsid protein of HCMV at the rim of the viral replication com-

92 partment, as well as an antagonizable effect of myosin Va on nuclear capsid localization to

93 the nuclear envelope (11).

94 These findings might argue for a role of nuclear F-actin in the trafficking of betaherpesvirus nucleocapsids to nuclear egress sites. However, direct evidence for active motility of capsids along nuclear filaments is missing. Recently, we developed a UL77-mGFP-tagged HCMV mutant that produces fluorescent nuclear capsids (12). We, therefore, set out to analyze the motility of HCMV nuclear capsids in relation to nuclear actin filaments by single particle tracking. While aiming at reproducing the induction of nuclear F-actin in HCMV-infected cells ex-

100 pressing LifeAct-NLS, we found that nuclear filament induction is dependent on the expres101 sion level and cellular localization of the utilized actin live-cell marker LifeAct. In our hands, 102 only cells with very high expression levels of LifeAct-mCherry-NLS presented nuclear fila103 ments, and reducing the expression levels by using a weaker promotor or utilizing a weakly 104 expressing cell population almost completely abrogated nuclear filament occurrence. Two 105 alternative fluorescent F-actin markers were unable to detect F-actin in HCMV-infected cells. 106 Using electron microscopy, we could only detect nuclear F-actin in infected cells expressing 107 mCherry-LifeAct-NLS, while in the absence of mCherry-LifeAct-NLS infected cells did not 108 show any. Finally, deleting the NLS abolished nuclear F-actin formation, which led us to con109 clude that nuclear actin induction in this system is an artifact of LifeAct-NLS overexpression. 110 In accordance, we did not find transport events along nuclear F-actin employing single particle 111 tracking. Instead, nuclear HCMV capsids showed diffusive motility with no indication for active 112 transport, which fits our measurements that HCMV infection also remodels the nuclear chro- 
113 matin structure to facilitate particle diffusion as described previously for members of the al-

114 phaherpesviruses. We, therefore, conclude that LifeAct-NLS serves as both an expression-

115 level-dependent inducer and detector of nuclear actin filaments in HCMV-infected cells, while HCMV infection itself does not induce nuclear actin in normal fibroblasts.

\section{Results}

HCMV infection does not induce nuclear actin filaments when LifeAct-NLS is expressed at medium levels

121 To test if nuclear HCMV capsids would use LifeAct-stainable nuclear filamentous actin for

122 transport, we first generated stable cell lines expressing LifeAct-mCherry-NLS similarly to an 123 approach reported earlier (10) based on both primary HFFs (data not shown) as well as 124 hTERT immortalized BJ cells. To visualize viral capsids, we used our recently described 125 UL77-tagged HCMV mutant (12). Since this UL77-mGFP fusion already occupied the GFP 126 channel, we exchanged GFP in the original LifeAct construct (RRID: Addgene_58467) with 127 the red-fluorescent mCherry, resulting in cells showing a homogenous nuclear LifeAct signal 128 which was slightly enriched in what seemed to be nucleoli. To our surprise, infection with 129 either WT, UL77-GFP, or another fluorescent virus, that produces UL32EGFP and 130 UL100mCherry, did not result in the formation of nuclear LifeAct-positive filaments in the vast 131 majority of cells. Only very rarely (2.33\% of infected cells) and only in cells expressing high 132 amounts of LifeAct, we found nuclear filaments (Fig. 1A/B).

134 The occurrence of nuclear actin filaments correlates with the expression level of Life- 
136 Interestingly, the already low frequency of nuclear filaments decreased even further with on-

137 going infection, such that it was not possible to detect any cells with nuclear filaments later 138 than 24 hours post infection (HPI) (Fig. 1B). We quantified nuclear LifeAct-mCherry-NLS in139 tensities and found that intensities decreased with ongoing infection (as indicated by nuclear 140 IE1 and nuclear pUL32-EGFP signals; Fig. 2A-C) to levels that were still easily detectable 141 using standard excitation levels but seemingly insufficient for filament formation.

142 Since the induction of nuclear actin assemblies appeared to be dependent on high expression 143 levels of LifeAct, we assumed that the promoter driving LifeAct-mCherry-NLS expression 144 might critically influence the appearance of these structures. In our expression system, we 145 used a spleen focus-forming virus- (SFFV-) promoter instead of a phosphoglycerate kinase146 (PGK-) promoter utilized in the original report (10), which led to the decrease in LifeAct147 mCherry-NLS signal intensity. We hypothesized that HCMV infection might interfere with ex148 pression from the SFFV promotor and therefore generated an alternative stable cell line ex149 pressing LifeAct-mCherry-NLS driven by the HCMV-Immediate early (HCMV-IE) promoter. 150 We expected this promotor to increase LifeAct-mCherry-NLS expression in HCMV-infected 151 cells. Indeed, we found that nuclear mCherry intensities increased when these cells were 152 infected with HCMV up to 48hpi as shown in Fig. $3 \mathrm{~A}$ and $\mathrm{B}$.

153 Importantly, we were now also able to detect nuclear filaments in infected cells although at 154 lower levels compared to the earlier report (10). However, LifeAct expression decreased with 155 progressing infection and at $72 \mathrm{hpi}$ reached levels that were comparable to mock-infected cells 156 (see histograms). This effect correlated with HCMV immediate early protein 1 (IE1) expres157 sion kinetics as also shown Figs $3 \mathrm{~A}$ and $\mathrm{B}$. We did not detect filaments in the mock-infected 158 cells, which is consistent with our previous experiments that showed no actin filaments stain- 
159 able with LifeAct or with phalloidin in non-infected cells. Upon infection, we were able to vis-

160 ualize nuclear actin assemblies in significant quantities (Figure 4). The high expression levels

161 of LifeAct-mCherry-NLS seemed to affect cell growth, as overall LifeAct-mCherry-NLS ex-

162 pression levels were reduced quickly after a few passages, which made it challenging to keep

163 expression levels constant in between experiments. Therefore, we show the results of exper-

164 imental replicates separately in Figure 4 A-D. As can be seen in Figure 4E, later passages

165 used in experiment 2 and 3 showed reduced LifeAct-mCherry-NLS expression compared to

166 earlier passages (experiment 1 and 4). LifeAct-mCherry-NLS expression in mock-infected

167 cells correlated well with the amount of nuclear filament induction after infection (compare

168 Fig. $4 A / D$ to $4 B / C)$, which indicates that filament induction is dependent on the LifeAct-

169 mCherry-NLS level. Also, LifeAct-mCherry-NLS intensities were exceptionally strong in cells

170 that showed filaments after infection compared to cells that did not as shown in Fig.4F, sup-

171 porting the idea that LifeAct-mCherry-NLS acts as a concentration-dependent inducer of nu-

172 clear F-actin assembly after HCMV infection.

173 Of note, cells that showed nuclear actin filaments $72 \mathrm{hpi} \mathrm{seemed} \mathrm{to} \mathrm{be} \mathrm{delayed} \mathrm{in} \mathrm{the} \mathrm{progres-}$ 174 sion of infection as indicated by the expression level of pUL32-EGFP (Fig. 5A, arrows), often 175 leading to the mutual exclusion of high LifeAct and pUL32 expression (Fig. 5B).

Cells not expressing LifeAct do not show Phalloidin-stainable nuclear actin assemblies during infection

179 Since our results suggested that LifeAct is an inducer and also detector of filamentous nuclear 180 actin at high expression levels, we next wanted to test if mock cells also show F-actin induc181 tion after HCMV infection. To do so, we first tested if the widely utilized fluorescent Phalloidin 182 can detect LifeAct-induced nuclear filaments. We infected BJ cells expressing LifeAct183 mCherry-NLS driven by the HCMV major immediate early promotor (MIEP) with HCMV-HB5- 
UL77-mGFP and fixed and stained them with fluorescent Phalloidin at $24 \mathrm{hpi}$. As shown in Fig. 6, LifeAct-positive nuclear filaments could also be detected with fluorescent Phalloidin. Importantly, however, we could not detect any nuclear actin structures in wt BJ cells infected with HCMV-HB5-UL77-mGFP. Interestingly, almost all (95\%) BJ-CMV-LifeAct-mCherry-NLS cells that showed nuclear filaments were infected as shown by IE1 staining, which indicates that the infection may be the blies had exceptionally high LifeAct expression levels. These findings point towards a concentration-dependent interference of LifeAct-NLS probes with nuclear actin polymerization dynamics which has been described previously $(13,14)$. We also observed that the infection rate in BJ-CMV-LifeAct-mCherry-NLS cells was significantly lower than in WT-BJ cells, which further indicates that nuclear LifeAct-mCherry-NLS inhibits HCMV replication.

\section{Deleting the NLS precludes LifeAct-dependent induction of nuclear actin assemblies}

198 Since our results argue for a cumulative effect of both LifeAct-mCherry-NLS expression and HCMV infection on nuclear actin availability and polymerization dynamics, we wanted to test if the NLS is the key to the induction of nuclear actin assemblies. We therefore created a cell line expressing LifeAct-mCherry without an NLS. As shown in Fig. 7, we did not detect nuclear actin filaments throughout infection in these cells, indicating that LifeAct-NLS-mediated shuttling of G-actin into the nucleus might play a role in the formation of nuclear filaments.

204 Off note, we sometimes observed thick cytoplasmic actin assemblies in infected cells ex205 pressing a high level of LifeAct, which might indicate that LifeAct can also induce similar as206 semblies in the cytoplasm (Fig. 7 arrow) when convoluted with other actin-modulating inter207 ferences like HCMV infection. 
An alternative live-cell actin probe fails to detect nuclear actin assemblies during in-

\section{fection}

211 Recent reports have shown that LifeAct fused to an NLS can interfere with nuclear actin dy-

212 namics (13) and some authors recommend nanobody technology as a better alternative. Es-

213 pecially an anti-nuclear-Actin Chromobody was referred to as minimally interfering with actin

214 dynamics and therefore more suited to assess actin filament formation in the nucleus (13,

215 15). We created a mixed cell clone expressing this nuclear-Actin Chromobody to have a spec-

216 trum of different expression levels to investigate nuclear actin formation upon infection with

217 HCMV. As shown in Figure 8, we did not find any nuclear filaments or other actin assemblies

218 in these cells.

\section{Nuclear capsids do not move along LifeAct positive nuclear actin filaments}

221 To functionally test if HCMV-induced nuclear actin filaments or actin assemblies are used for directed nuclear capsid transport, we used an HCMV mutant that produces fluorescent capsids (HCMV UL77-GFP (12)). We infected BJ cells stably expressing LifeAct-mCherry-NLS with HCMV UL77-GFP and imaged cells 72 hpi post infection. As described above, infected moved in a random-walk-like fashion through the nucleus.

228 To determine the motility mode of HCMV nuclear capsids at a quantitative level, we used

229 single particle tracking as done previously for alphaherpesviruses (6). To this end, we infected

230 BJ cells with HCMV UL77-GFP and imaged the cells shortly after first capsids appeared at

$23172 \mathrm{hpi}$. To determine particle motility modes, we optimized our established particle tracking 232 workflow (7) using a batch-adopted version of Trackmate (16) (see Materials and Methods). 
233 Custom Matlab scripts (see Materials and Methods) allowed us to convert the data and feed

234 it into MSDanalyzer (17) as described earlier (7).

236 We were able to extract a significantly larger number of particle tracks from our data using

237 this approach, increasing the statistical validity of our analysis. In line with our visual assessment, our quantification of more than 17000 single tracks longer than 1 second (20 frames) showed that HCMV nuclear capsids do not engage in directed motility, but show slight subdiffusion with an anomalous diffusion exponent $\alpha$ of 0.74 in the nucleoplasm over short timescales (Fig. 10). This result is a little lower than our previously published data for HSV-1 and PRV. A calculation of corral size, performed as in (7), revealed a corral size of approximately

$243700 \mathrm{~nm}$ and indicates that also HCMV remodels the nuclear structure very similarly to HSV-1 244 and PRV, which in turn would allow nuclear capsids to cross large areas of the nuclear space 245 by diffusion.

Electron microscopy could not to detect nuclear actin bundles in normal infected cells

248 To determine if normal infected cells that do not express LifeAct-NLS show any form of nu249 clear filaments that cannot be detected using fluorescent probes, we applied electron microscopy as an additional tool. We seeded BJ-CMV-LifeAct-mCherry-NLS cells on sapphire discs and infected them with HCMV. The infected cells were checked by live-cell fluorescence microscopy for the appearance of nuclear actin structures and subsequently high-pressure-frozen and processed for transmission electron microscopy. Indeed, we found thick bundles of

254 nuclear filaments in a fraction of cells expressing LifeAct-mCherry-NLS (Fig. 11A-B, S3).

255 However, in line with our previous experiments, we could not detect nuclear filaments in cells 256 that expressed LifeAct-mCherry-NLS at low levels. The bundles showed strong similarity to 
previously published nuclear actin bundles induced by overexpression of actin mutants (18).

258 The bundles appeared more often close to the nuclear envelope, but we did not find evidence 259 for filaments connecting the nuclear envelope to a nascent central viral replication compart260 ment as proposed earlier (10).

\section{Discussion}

263 In this study, we investigated nuclear actin filament formation after HCMV infection. We found

264 that only infection of cells highly overexpressing LifeAct-mCherry-NLS leads to nuclear fila265 ment formation. Cells expressing lower levels of nuclear LifeAct-mCherry-NLS (which was 266 still easily detectable) did not show filament induction. Furthermore, Phalloidin failed to detect nuclear filaments in infected cells that do not express LifeAct-mCherry-NLS, but was able to mark filaments in infected, LifeAct-overexpressing cells. We, therefore, conclude that LifeActmCherry-NLS is a concentration-dependent inducer of nuclear actin structures in HCMV-infected cells. Our results are supported by a previous study that showed that nuclear actin

271 filaments could be induced by overexpression of NLS-actin fusion proteins (18) as well as a 272 more recent study that describes the interference of LifeAct-NLS probes with nuclear actin 273 dynamics, resulting in the formation of nuclear actin assemblies (13).

274 For this reason, a recent review recommended the use of alternative markers to assess the 275 nuclear actin dynamics in living cells such as an anti-actin-chromobody (15). This probe failed 276 to detect any filamentous actin structures in normal, HCMV-infected cells. In line with these 277 results, TEM analysis showed large nuclear bundled filamentous structures in a subpopula278 tion of HCMV-infected cells that expressed high levels of LifeAct-NLS. These structures are 279 easily detectable in EM if they appear in normal infected cells. However, we could not detect 
any of those structures in normal infected BJ cells. To our knowledge, there is also no EMbased evidence of nuclear actin bundles in HCMV-infected cells in the literature. In our hands, capsid formation and nuclear filament induction appeared to be almost mutually exclusive, which indicates that overexpression of the LifeAct-mCherry-NLS probe is detrimental for the progression of viral infection. For this reason, we could visualize capsid movement in the presence of actin assemblies in a few cells only. Still, capsid movement was independent of actin assemblies and was indistinguishable from capsid motility in fibroblasts not expressing LifeAct. Single particle tracking of HCMV nuclear capsids showed that diffusion is the main motility mode, and confirmed our previous results for $\mathrm{HSV}-1$ and PRV $(6,7)$, indicating that nuclear capsid diffusion might be a conserved motility mode to cross the nucleoplasm. Support for this conclusion also comes from two recent studies in which the au-

291 thors first show that HSV-1 induces egress channel formation through the marginalized chro292 matin (8), which could represent the motility spaces that can be measured by tracking nuclear 293 capsids ((7) and Fig. 10). Secondly, computational simulations using our experimentally de294 termined diffusion coefficient suggest that these egress channels allow capsid translocation 295 to the nuclear membrane by diffusion within minutes (9).

296 The question remains which molecular mechanism induces filament formation in LifeAct-NLS297 expressing cells. Since a LifeAct construct without the NLS sequence was unable to induce 298 actin structures in the nucleus of infected cells, we hypothesize that LifeAct-NLS increases 299 the concentration of monomeric G-actin in the nucleus. Infection-induced disruption of cyto300 plasmic F-actin (19) might increase the available G-actin pool, which could result in exceeding 301 a nuclear concentration threshold at which filament formation occurs (18). While Wilkie et al. 302 (10) did not detect an increase of nuclear actin monomers in infected cells, they did not com303 pare cells with and without the LifeAct-GFP-NLS construct. It is therefore currently not clear 
304 if increased amounts of monomeric G-actin get transported into the nucleus by LifeAct-NLS during HCMV infection.

The role of actin in the nucleus is a matter of current debate, and intimately connected to a discussion about the strengths and weaknesses of the actin probes used $(14,15,20-25)$. A variety of roles has been described for nuclear actin in recent years, ranging from gene regulation to structural organization (24). Widely used probes like LifeAct can interfere with nuclear actin polymerization, and these caveats must be taken into account when assessing the

311 biological role of nuclear actin filaments $(13,20)$. The reasons for the artifacts are often un-

312 clear. However, it is likely that nuclear-targeted probes alter the nuclear concentration of actin

313 monomers by shuttling G-actin into the nucleus $(13,14)$, which is also supported by our re314 sults. Probe-induced nuclear filament formation has not only been described for LifeAct-NLS, 315 but also for UTR261 and UTR230-NLS fusions $(13,14)$.

316 Based on our results we conclude that HCMV does not induce large-scale nuclear actin as317 semblies, and that nuclear capsid motility is not dependent on large actin tracks. However, 318 we cannot exclude that more subtle and maybe transient actin assemblies play a role in nu319 clear morphogenesis events. Possible targets might be genome encapsidation and release 320 of capsids from the replication compartment. Future studies will have to be carefully designed 321 to examine the role of actin in nuclear herpesvirus morphogenesis while omitting the known 322 pitfalls of nuclear actin probes.

\section{Materials and methods}

325 Cells and viruses. BJ-5ta hTERT-immortalized human fibroblasts were licensed from ATCC 326 (CRL-4001) and cultivated in Dulbecco's Modified Eagles Medium Glutamax ${ }^{\circledR}$ (Thermofisher) 
with 20\% Medium 199 (Earles Salts) (Thermofisher), 10\% FBS superior (Merck), $0.8 \mathrm{mM}$ sodium pyruvate (Thermofisher) and $1 \mu \mathrm{g} / \mathrm{ml}$ Hygromycin (Invivogen).

BAC-derived AD169-based HCMV-HB5-UL77-mGFP is described in reference (12). HCMV-

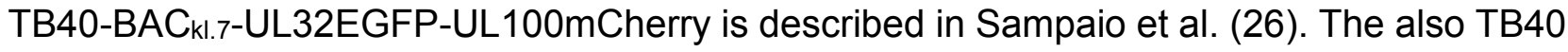
based HCMV-UL32-EGFP is reported in (27).

pSFFV and pCMV-driven LifeAct-2XNLS expression constructs. The lentiviral plasmid insert reflecting the pEGFP-C1_LifeAct-EGFP_2XNLS construct (20). (pEGFP-C1 LifeActEGFP-2XNLS was a gift from Dyche Mullins (RRID: Addgene_58467). We also introduced an upstream 5' Avrll site to facilitate cloning. The resulting sequence was inserted into LeGO338 iC2 (28), thereby replacing the original IRES-mCherry sequence of LeGO-iC2 between 339 BamHI and BsrGI. To generate LeGO_CMV_LifeAct-mCh-2XNLS, we replaced the SFFV promoter of the original gene expression cassette by the HCMV major immediate early promoter sequence via standard PCR-based cloning using Nhel and Avrll. Correct sequences of both expression constructs were confirmed by Sanger sequencing.

Immunofluorescence. For quantification of LifeAct or chromobody intensities during HCMV infection, cells were cultivated in BJ-Medium and seeded on Ibidi glass bottom $\mu$-Dishes coated with Fibronectin 1:100 in Dulbecco's phosphate buffered saline (D-PBS, Sigma-Aldrich). After infection with HCMV-TB40-BACkl.7-UL32EGFP-UL100mCherry or HCMVUL32GFP (gift from Lüder Wiebusch) at a multiplicity of infection (MOI) of 10. Cells were fixed 349 with $4 \%$ paraformaldehyde (Science Services) in D-PBS at $24 \mathrm{hpi}$, 48hpi, and $72 \mathrm{hpi}$. For im350 munofluorescence (IF) staining, the cells were permeabilized with $0.1 \%$ TritonX100 in D-PBS, 
blocked with 3\% Bovine Serum Albumin (BSA) in D-PBS, and subsequently stained with a primary murine anti-IE1 antibody and a secondary Alexa 647 Goat anti-mouse antibody (Thermofisher). Nuclei were additionally stained with Hoechst 33342 (Thermofisher). For Phalloidin staining, BJ-WT and BJ-CMV-LifeAct-mCherry-NLS were infected with HCMVHB5-UL77-mGFP at an MOI of 1 for $24 \mathrm{~h}$ and subsequently fixed and stained with Alexa-488Phalloidin (Thermofisher) and Hoechst 33342 (Thermofisher).

Microscopy was performed with a Nikon spinning disc system consisting of a Yokogawa W2 and two Andor iXON888 cameras using NIS-Elements for image acquisition. A Nikon 100x 1.49 NA Apo-TIRF objective was used resulting in $130 \mathrm{~nm}$ pixel size. The system was equipped with standard 405, 488, 561, $640 \mathrm{~nm}$ laser lines and corresponding filter sets. For quantification, 5x5 image tiles were acquired, resulting in a 666x666 $\mu \mathrm{m}$ captured area.

364 Image analysis was performed with an ImageJ macro and a python Jupyter notebook (both 365 available on github through https://github.com/QuantitativeVirology/FIJI-Segmentor-Macro and https://github.com/QuantitativeVirology/FIJI-Measurement-Analyzer). Nuclei segmentation was done in ImageJ using the Hoechst channel creating regions of interest (ROIs), which were subsequently used to measure the signal intensities in the other channels of interest. Resulting mean signal intensities were processed in Python. The number of cells containing filaments was counted using the manual cell counter plugin from ImageJ. Statistical analysis was done with GraphPad PRISM.

373 Single particle tracking. For tracking of single viral particles in cell nuclei, BJ-WT cells were 374 cultivated in BJ-Medium and infected with HCMV-HB5-UL77-mGFP (MOI of 1.5). 72hpi vid375 eos of living cells were acquired with 21.45 frames per second (fps) at $37^{\circ} \mathrm{C}$ with $5 \% \mathrm{CO}_{2}$. 
376 Single nuclei were cropped, and capsids were tracked with the Fiji plugin Trackmate by Tine-

377 vez et al. (16), using a custom batch analysis plugin (available on github through https://github.com/QuantitativeVirology/Trackmate_Batch). The resulting XML files were analyzed using custom Matlab scripts (available on github through https://github.com/QuantitativeVirology/Matlab-Trackmate-MSD), which in turn make use of the Matlab class "Mean square displacement analysis of particle trajectories", also from Tinevez and colleagues (17). Visualization of the results was also done with Matlab.

Electron Microscopy. For structural analysis of nuclear actin assemblies, Sapphire discs (M. sion in soapy water and sonication for 10 minutes. Afterward, the discs were washed in $>99 \%$ Ethanol (Roth) twice by sonication for 10 minutes each and plasma cleaned in a Quorum Q150 plus (Quorum Technologies Ltd, UK) machine for 120 seconds and subsequently coated with a thin film of carbon through carbon cord evaporation. The discs were dried over390 night at $60^{\circ} \mathrm{C}$ and kept at that temperature until shortly before use.

391 BJ-CMV-LifeAct-mCherry-NLS cells were cultivated in BJ-Medium and seeded on the previ392 ously prepared sapphire discs. On the following day, the cells were infected with HCMV393 TB40e-UL32EGFP-UL100mCherry at an MOI of 10. After 24 hours cells were imaged through 394 live-cell spinning disc microscopy to check for LifeAct-induced filaments. Afterwards, cells were high-pressure frozen as described in (29).

396 For freeze-substitution, sapphire discs were incubated in $-90^{\circ} \mathrm{C}$ pre-cooled freeze substitution 397 medium consisting of $0.2 \%$ Osmium tetroxide (Science Services), $0.1 \%$ Uranyl acetate 398 (Merck) and 5\% water in Acetone (Merck) overnight at $-90^{\circ} \mathrm{C}$ in an Arctiko DP-80 cryo porter 
399 (Arctiko, Denmark) and subsequently thawed by stopping the cooling and leaving the machine

400 to warm to room temperature.

401 The freeze-substituted samples were subsequently embedded in Epon and cut into ultrathin 402 50nm slices using a Leica Ultracut microtome (Leica, Germany). The slices were transferred 403 to copper mesh grids, post-stained in saturated Uranyl acetate in $70 \%$ Ethanol (Roth) for 7 404 minutes and subsequently imaged using an FEI Tecnai F20 electron microscope (Ther405 mofisher, USA).

406

\section{Acknowledgments}

408 We thank Christian Sinzger (University of Ulm, Germany) for the gift of dual-color HCMV 409 through Jens von Einem (University of Ulm, Germany). We also thank Lüder Wiebusch (Char410 ite Berlin) for HCMV-UL32GFP. pEGFP-C1 LifeAct-EGFP-2XNLS was a gift from Dyche Mul411 lins through Addgene (plasmid \# 58467). LeGO-iC2 was a kind gift of Kristoffer Riecken and 412 Boris Fehse (UKE Hamburg). This study was funded through a Wellcome Trust collaborative 413 award to JBB (209250/Z/17/Z) and KG (209250/Z/17/Z). The Heinrich Pette Institute, Leibniz 414 Institute for Experimental Virology is supported by the Free and Hanseatic City of Hamburg 415 and the Federal Ministry of Health. MM and KG are supported by the Cluster of Excellence 416 RESIST (EXC 2155), Hannover Medical School, Hannover, Germany.

\section{References}

421 velope Proteins in a Stable Cytoplasmic Compartment during Human Cytomegalovirus Rep422 lication: Characterization of a Potential Site of Virus Assembly. J Virol 74:975-986. 
424 2. Sanchez V, Sztul E, Britt WJ. 2000. Human Cytomegalovirus pp28 (UL99) Localizes to a

425 Cytoplasmic Compartment Which Overlaps the Endoplasmic Reticulum-Golgi-Intermediate 426 Compartment. J Virol 74:3842-3851.

3. Mettenleiter TC, Müller F, Granzow H, Klupp BG. 2013. The way out: what we know and do not know about herpesvirus nuclear egress. Cell Microbiol 15:170-178.

4. Forest T, Barnard S, Baines JD. 2005. Active intranuclear movement of herpesvirus capsids. Nature Cell Biology 7:429-431.

5. Feierbach B, Piccinotti S, Bisher M, Denk W, Enquist LW. 2006. Alpha-Herpesvirus Infection Induces the Formation of Nuclear Actin Filaments. PLoS Pathogens 2:e85.

6. Bosse JB, Virding S, Thiberge SY, Scherer J, Wodrich H, Ruzsics Z, Koszinowski UH, 438 Enquist LW. 2014. Nuclear Herpesvirus Capsid Motility Is Not Dependent on F-Actin. Mbio 439 5:e01909-14.

441 7. Bosse JB, Hogue IB, Feric M, Thiberge SY, Sodeik B, Brangwynne CP, Enquist LW.

442 2015. Remodeling nuclear architecture allows efficient transport of herpesvirus capsids by

443 diffusion. Proceedings of the National Academy of Sciences 112:E5725-E5733.

445 8. Myllys M, Ruokolainen V, Aho V, Smith EA, Hakanen S, Peri P, Salvetti A, Timonen J, 446 Hukkanen V, Larabell CA, Vihinen-Ranta M. 2016. Herpes simplex virus 1 induces egress 
447 channels through marginalized host chromatin. Scientific Reports 6:28844.

449 9. Aho V, Myllys M, Ruokolainen V, Hakanen S, Mäntylä E, Virtanen J, Hukkanen V, Kühn

450 T, Timonen J, Mattila K, Larabell CA, Vihinen-Ranta M. 2017. Chromatin organization regu-

451 lates viral egress dynamics. Sci Rep-uk 7:3692.

10. Wilkie AR, Lawler JL, Coen DM. 2016. A Role for Nuclear F-Actin Induction in Human

Cytomegalovirus Nuclear Egress. mBio 7:e01254-16.

12. Borst E, Bauerfeind R, Binz A, Stephan T, Neuber S, Wagner K, Steinbrück L, Sodeik B,

460 Roviš T, Jonjić S, Messerle M. 2016. The Essential Human Cytomegalovirus Proteins

461 pUL77 and pUL93 Are Structural Components Necessary for Viral Genome Encapsidation. J Virol 90:5860-5875.

13. Du J, Fan Y, Chen T, Feng X. 2015. Lifeact and Utr230 induce distinct actin assemblies

14. Belin BJ, Mullins DR. 2013. What we talk about when we talk about nuclear actin. Nucl

Austin Tex 4:291-7.

470 15. Melak M, Plessner M, Grosse R. 2017. Actin visualization at a glance. J Cell Sci 471 130:jcs.189068. 
473 16. Tinevez J-Y, Perry N, Schindelin J, Hoopes GM, Reynolds GD, Laplantine E, Bednarek SY, Shorte SL, Eliceiri KW. 2017. TrackMate: An open and extensible platform for singleparticle tracking. Methods San Diego Calif 115. raël A, Laplantine E. 2014. TNF and IL-1 exhibit distinct ubiquitin requirements for inducing NEMO-IKK supramolecular structures. J Cell Biology 204:231-245.

18. Kokai E, Beck H, Weissbach J, Arnold F, Sinske D, Sebert U, Gaiselmann G, Schmidt V, Walther P, Münch J, Posern G, Knöll B. 2014. Analysis of nuclear actin by overexpression of wild-type and actin mutant proteins. Histochemistry and Cell Biology 141:123-135.

19. Jones N, Lewis J, Kilpatrick. 1986. Cytoskeletal disruption during human cytomegalovirus infection of human lung fibroblasts. Eur J Cell Biol 41:304-12.

20. Belin BJ, Cimini BA, Blackburn EH, Mullins DR. 2013. Visualization of actin filaments and monomers in somatic cell nuclei. Molecular Biology of the Cell 24:982-994. ized actin in live cells with LifeAct fused to fluorescent proteins. Nat Cell Biol 18:676-683. forms of stress-induced twisted f-actin. Nat Methods 6:nmeth0509-317. 
497 23. Riedl J, Crevenna AH, Kessenbrock K, Yu J, Neukirchen D, Bista M, Bradke F, Jenne

498 D, Holak TA, Werb Z, Sixt M, Wedlich-Soldner R. 2008. Lifeact: a versatile marker to visual499 ize F-actin. Nat Methods 5:605-607.

24. Viita T, Vartiainen MK. 2016. From Cytoskeleton to Gene Expression: Actin in the Nucleus. Handb Exp Pharmacol.

25. Flores LR, Keeling MC, Zhang X, Sliogeryte K, Gavara N. 2019. Lifeact-GFP alters Factin organization, cellular morphology and biophysical behaviour. Sci Rep-uk 9:3241.

26. Sampaio K, Jahn G, Sinzger C. 2013. Virus-Host Interactions, Methods and Protocols. Methods Mol Biology Clifton N J 1064:201-209. beled with Green Fluorescent Protein for Live Analysis of Intracellular Particle Movements. J Virol 79:2754-2767.

514 28. Weber K, Bartsch U, Stocking C, Fehse B. 2008. A Multicolor Panel of Novel Lentiviral 515 "Gene Ontology" (LeGO) Vectors for Functional Gene Analysis. Mol Ther.

517 29. Höhn K, Sailer M, Wang L, Lorenz M, Schneider ME, Walther P. 2011. Preparation of 518 cryofixed cells for improved 3D ultrastructure with scanning transmission electron tomogra519 phy. Histochem Cell Biol 135:1-9. 


\section{Figure legends}

521 Figure 1. A very small fraction of cells shows nuclear F-Actin after HCMV infection. BJ-

522 SFFV-LifeAct-mCherry-NLS cells were infected with HCMV-TB40/e-UL32EGFP-

523 UL100mCherry, fixed at 24hpi and stained for DNA (Hoechst) and HCMV-IE (Anti-IE1). (A)

524 Representative image of very rare nuclear actin filaments. Scale bar indicates $10 \mu \mathrm{m}$. (B)

525 Quantification of the filament rates. Large tiles spanning $0.75 \times 0.75 \mu \mathrm{m}$ were acquired and 526 quantified in ImageJ and Python using scripts (see Materials and Methods). Filaments were 527 manually counted. Means of three independent replicates are shown. Bars indicate standard 528 deviations.

Figure 2: LifeAct-mCherry-NLS signal diminishes with ongoing infection. BJ-SFFV-LifeAct-mCherry-NLS cells were infected with HCMV-TB40/e-UL32EGFP-UL100mCherry, fixed at the indicated time points and stained for DNA (Hoechst) and HCMV-IE (Anti-IE1). pUL32EGFP serves as a marker for late gene expression. (A) LifeAct-mCherry-NLS signal intensity

534 drops with ongoing infection. Scale bars indicate $10 \mu \mathrm{m}$. (B) Quantification of subcellular Life535 Act vs. IE signal intensities at 24, 48 and 72hpi using automated microscopy image analysis. 536 (C) LifeAct intensity over time. For comparison, all image intensities are scaled to the same 537 level in Fig. 2 and 3. Scale bars indicate $10 \mu \mathrm{m}$.

Figure 3. Changing the promoter of the LifeAct expression constructs alters expres-

540 sion dynamics in infection. BJ-CMV-LifeAct-mCherry-NLS cells were infected with HCMV541 TB40/e-UL32EGFP-UL100mCherry, fixed at the indicated time points and stained for DNA 542 (Hoechst) and HCMV-IE (Anti-IE1). pUL32-EGFP serves as a marker for late gene expres- 
543 sion. (A) LifeAct-mCherry-NLS signal intensity drops with ongoing infection. Scale bars indi-

544 cate $10 \mu \mathrm{m}$. (B) Quantification of subcellular LifeAct vs. IE signal intensities at 24, 48 and

$54572 \mathrm{hpi}$ by automated microscopy image analysis. (C) LifeAct intensity over time. All image 546 intensities are scaled to the same level in Fig. 2 and 3 for comparison. Scale bars indicate 10 $547 \mu \mathrm{m}$

Figure 4. Induction of nuclear filamentous structures is dependent on LifeAct expression. BJ-CMV-LifeAct-mCherry-NLS cells were infected with HCMV-TB40/e-UL32EGFPUL100mCherry, fixed at the indicated time points and stained for DNA (Hoechst) and HCMVIE (Anti-IE1). (A-D) Rate of LifeAct nuclear filaments in infected IE-1-positive cells. Four replicates with different base-line expression levels of LifeAct are shown as quantified in (E). (F) Difference in LifeAct-mCherry-NLS signal intensity in the cells with filaments $(+)$, compared to those without (-).

Figure 5. Strong LifeAct-mCherry-NLS expression and filaments induction block progress of infection. BJ-CMV-LifeAct-mCherry-NLS cells were infected with HCMV-TB40/eUL32EGFP-UL100mCherry, fixed at 72hpi and stained for DNA (Hoechst) and HCMV-IE (Anti-IE1). pUL32-EGFP serves as a marker for late gene expression. (A) Cells that show pUL32-EGFP expression have very little LifeAct-mCherry-NLS signal. Scale bar indicates 20 $\mu \mathrm{m}$. (B) Scatter plot of nuclear LifeAct-mCherry-NLS vs. pUL32-EGFP signal compared to the nuclear mCherry signal. and BJ-CMV-LifeAct-mCherry-NLS were infected with HCMV-HB5-UL77-mGFP at an MOI of 56710 for 24 hours, fixed, and stained for IE1, as well as with Alexa-488-Phalloidin. The arrow 
568 indicates a representative BJ-CMV-LifeAct-mCherry-NLS cell in which the same nuclear actin

569 structures are stained by both LifeAct as well as by Phalloidin. Scale bar is $10 \mu \mathrm{m}$.

Figure 7. A LifeAct-mCherry fusion missing the NLS does not induce nuclear filaments. loidin, and BJ-CMV-LifeAct-mCherry-NLS are shown. Scale bars indicate $10 \mu \mathrm{m}$.

Figure 8. A nuclear anti-actin chromobody did not to detect nuclear actin structures.

(A) BJ cells stably expressing a nuclear anti-actin chromobody were infected with HCMV-

581 Representative images illustrating that the chromobody does not detect any nuclear actin filaments in infected cells. Scale bars indicate $20 \mu \mathrm{m}$. (B) Quantification of filament occurrence as detected by the chromobody. One representative experiment out of 3 replicates is shown.

Figure 9. Capsids move independently of nuclear actin structures. BJ-CMV-LifeActmCherry-NLS cells were infected with HCMV-HB5-UL77-mGFP and imaged live at 72hpi at frames shows diffusive green clouds of particle location. The video is available as supplementary video S1. Scale bar indicates $10 \mu \mathrm{m}$. 
591 Figure 10. Single particle tracking reveals diffusion as the major nuclear capsid motility

592 mode. WT BJ cells were infected with HCMV-HB5-UL77-mGFP and imaged live at 72hpi at

593 a frame rate of $21.45 \mathrm{fps}$, and capsids were tracked using a custom batch version of Track-

594 mate. Tracks were subsequently analyzed utilizing MSD Analyzer, and the diffusion exponent

595 alpha (A), as well as the average corral size using an MSD plot (B), were extracted. The 596 saturation of the MSD curve at about 0.08 indicates a chromatin corral diameter of about 700 $597 \mathrm{~nm}$

Figure 11. Electron microscopy only reveals thick filamentous bundles in infected cells

expressing LifeAct-mCherry-NLS. BJ or BJ-CMV-LifeAct-mCherry-NLS cells were infected with HCMV-TB40/e-UL32EGFP-UL100mCherry, high-pressure-frozen at 24hpi and freezesubstituted. (A) Bundled, filamentous actin structures can be visualized in the nuclei of in-

603 fected cells expressing LifeAct-mCherry-NLS. Bundles only appeared in a fraction of the ex604 amined cells and in higher frequency closer to the nuclear envelope (see also supplementary 605 Figure S3). (B) Two cells without apparent nuclear actin structures visible for comparison. For 606 scale bar length see picture.

607

\section{Supplementary Material}

Video S1 and S2. Single viral particles are moving through the nucleus of an infected

611 cell. In these videos, the nuclei of BJ-CMV-LifeAct-mCherry-NLS cell infected with HCMVHB5-UL77-mGFP (MOI of 1.5) are shown at 72hpi. They represent rare examples in which nuclear actin assemblies and viral capsids are visible in the nucleus. Viral particles do not 
614 show obvious movement along the filaments. Instead, they move in a random-walk like be615 havior through the nucleoplasm.

617 Figure S3. Electron micrograph of a nucleus from an infected, high-pressure frozen

618 cell. Lower section of an BJ-CMV-LifeAct-mCherry-NLS cell infected with HCMV-TB40/e-

619 UL32EGFP-UL100mCherry which was high-pressure-frozen at 24hpi and subsequently 620 freeze-substituted. This cell is an example of the high density of actin bundles adjacent to the

621 nuclear envelope. (A/B) Details of the bundles shown. Scales are indicated in the pictures. 
bioRxiv preprint doi: https://doi org/101101/641266; this version posted May 24 2019. The copyright holder for this preprint (which was not certified by peer review) is the author/funder, who has granted bioRxiv a license to display the preprint in perpetuity. It is made available under aCC-BY-NC-ND 4.0 International license.

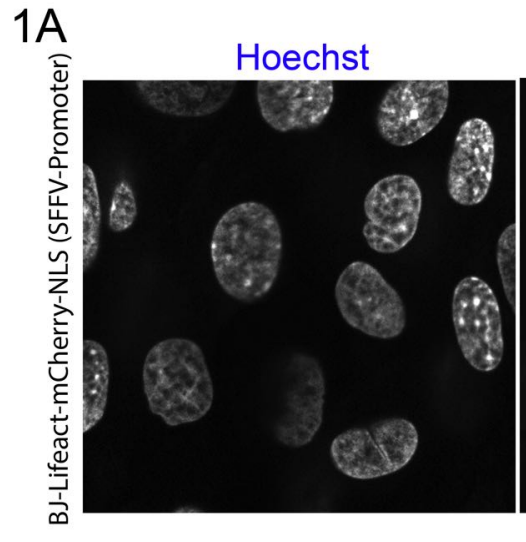

$1 \mathrm{~B}$

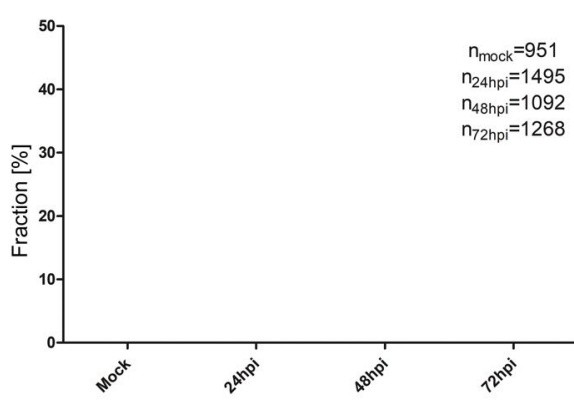

Lifeact-mCherry-NLS
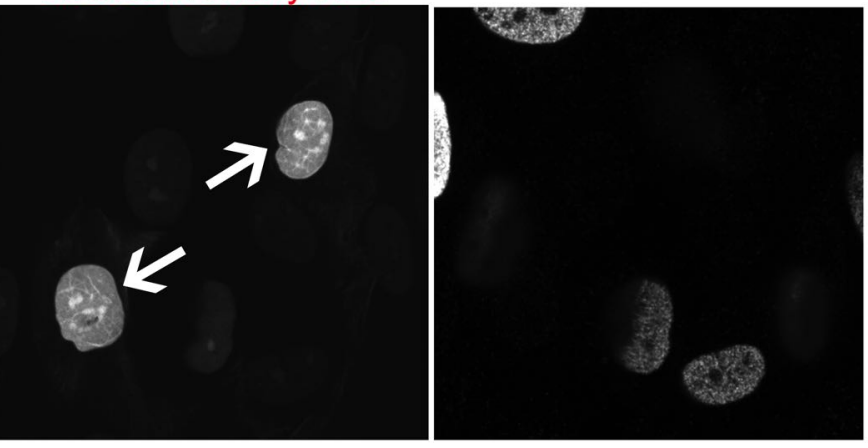

Rates of Filaments in Cells

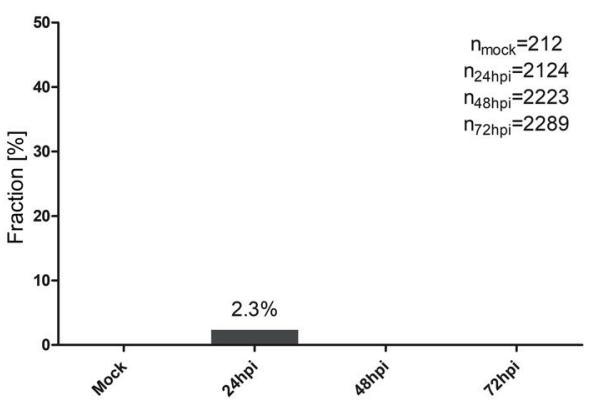

Merge

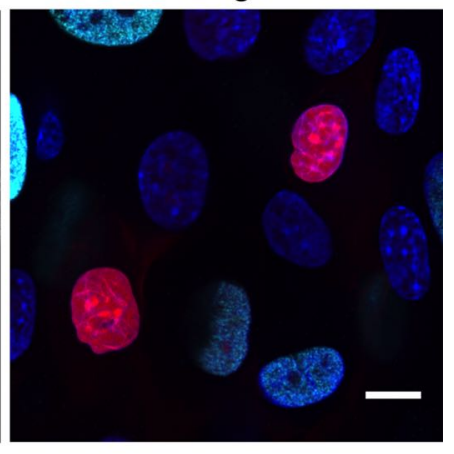

Mean Filament Rate over 3 Replicates: 0.9\%

$\mathrm{n}_{\text {Mock }}=1462$

$\mathrm{n}_{24 \mathrm{hpi}}=769$

$\mathrm{n}_{48 \mathrm{hpi}}=883$

$\mathrm{n}_{48 \mathrm{hpi}}=883$
$\mathrm{n}_{72 \mathrm{hpi}}=623$

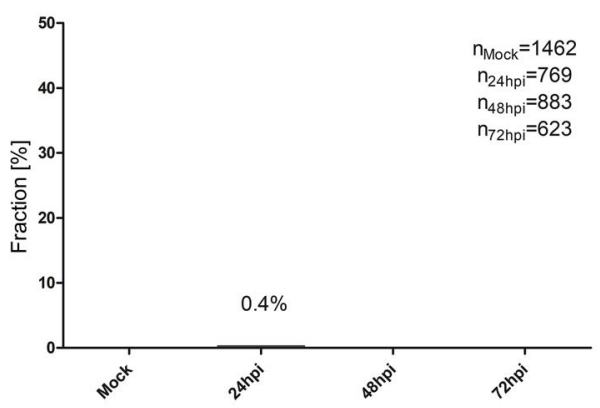


bioRxiv preprint doi: https://doi org/10.1101/641266; this version posted May 24 2019. The copyright holder for this preprint (which was

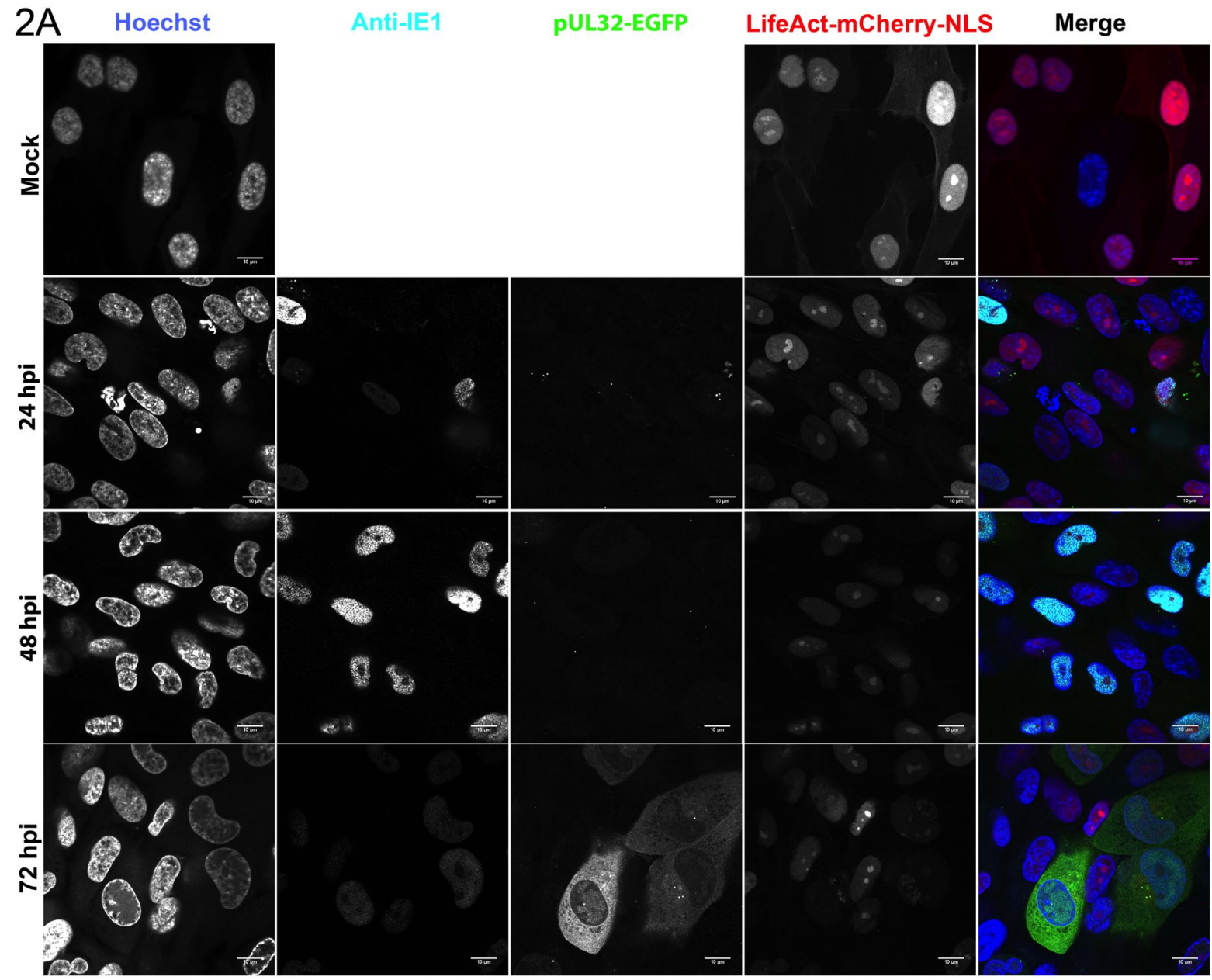

2B

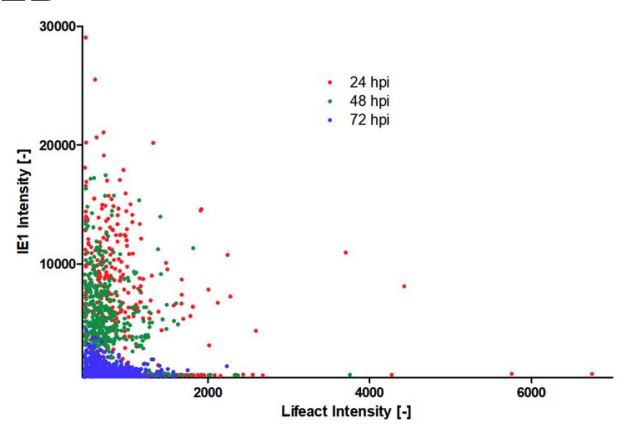

$2 \mathrm{C}$

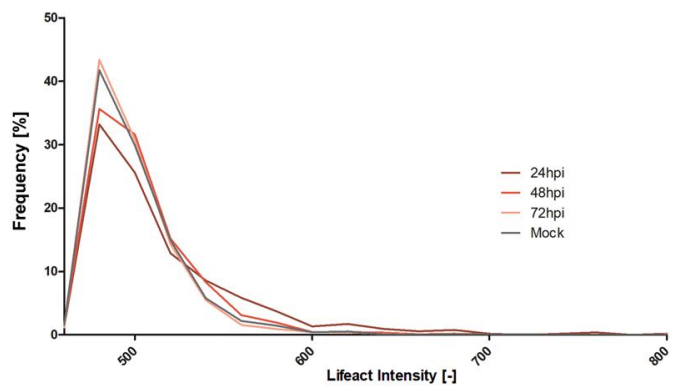


bioRxiv preprint doi: https://doi org/10.1101/641266; this version posted May 24 2019. The copyright holder for this preprint (which was not certified by peer review) is the author/funder, who has granted bioRxiv a license to display the preprint in perpetuity. It is made available under aCC-BY-NC-ND 4.0 International license.
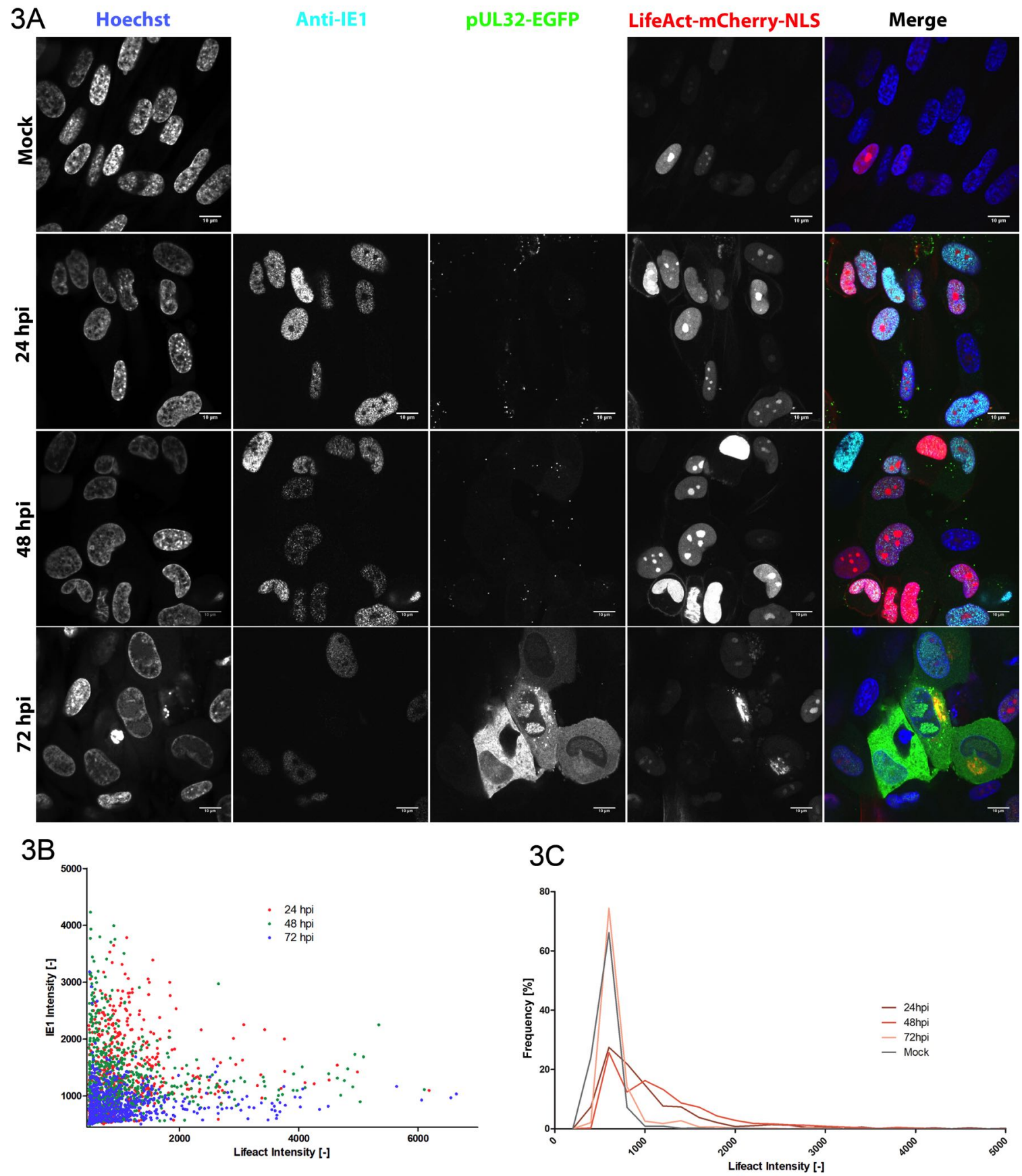


\section{Rate of nuclear filaments in BJ-CMV-Lifeact-mCherry-NLS}
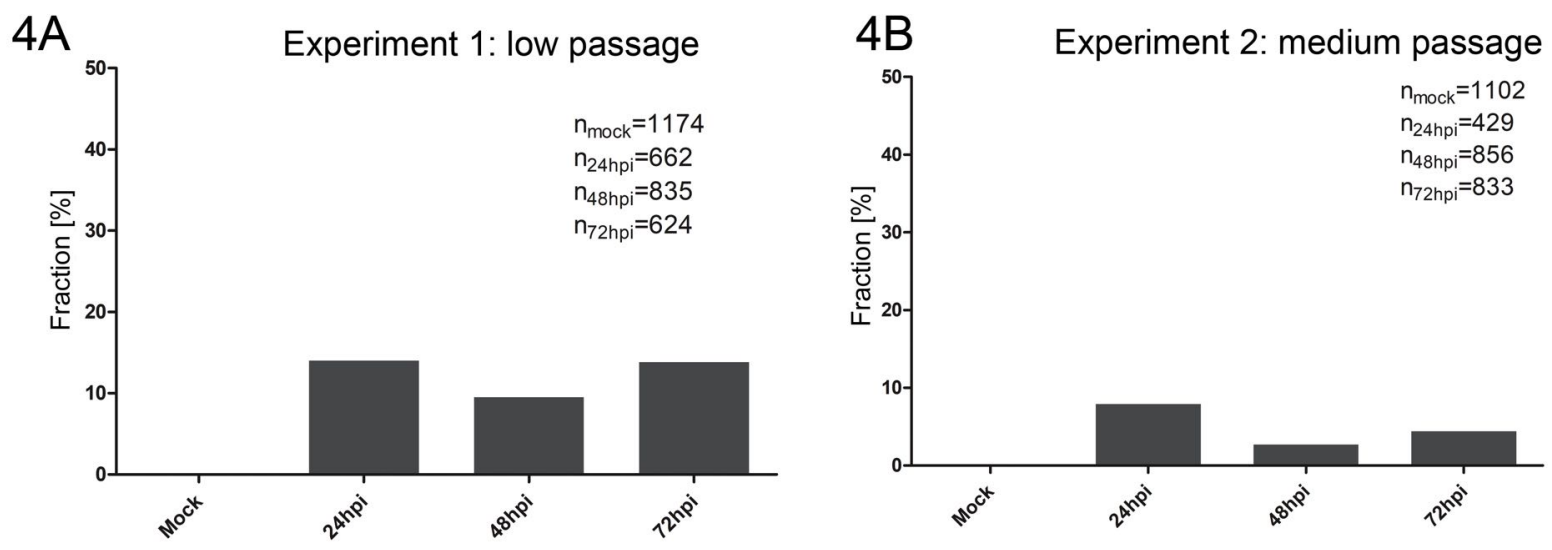

$4 \mathrm{C}$

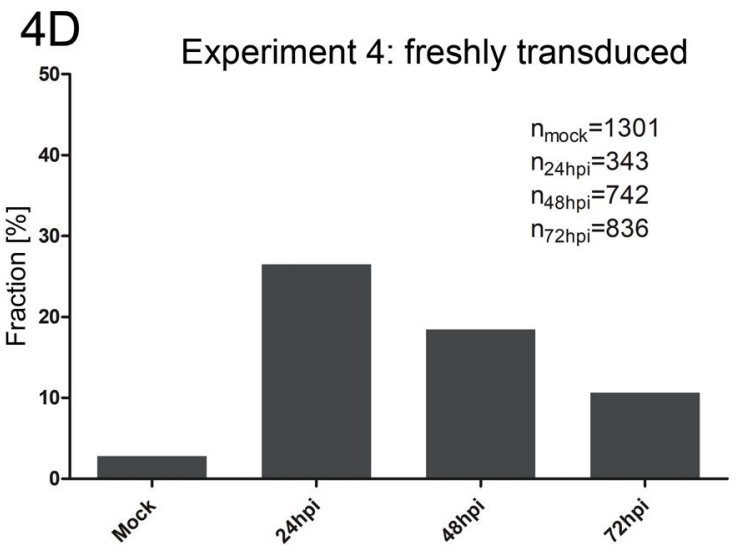

$4 \mathrm{E}$

Experiment 3: high passage

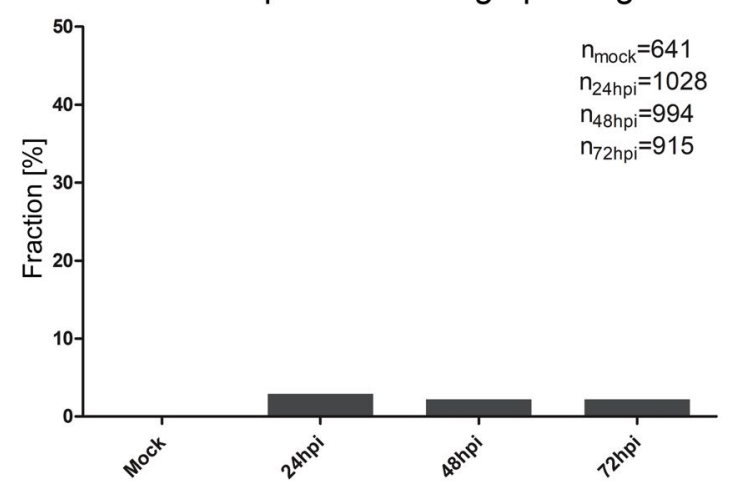

4F

4F Llfeact intensity in cells $w /$ filaments vs. w/o
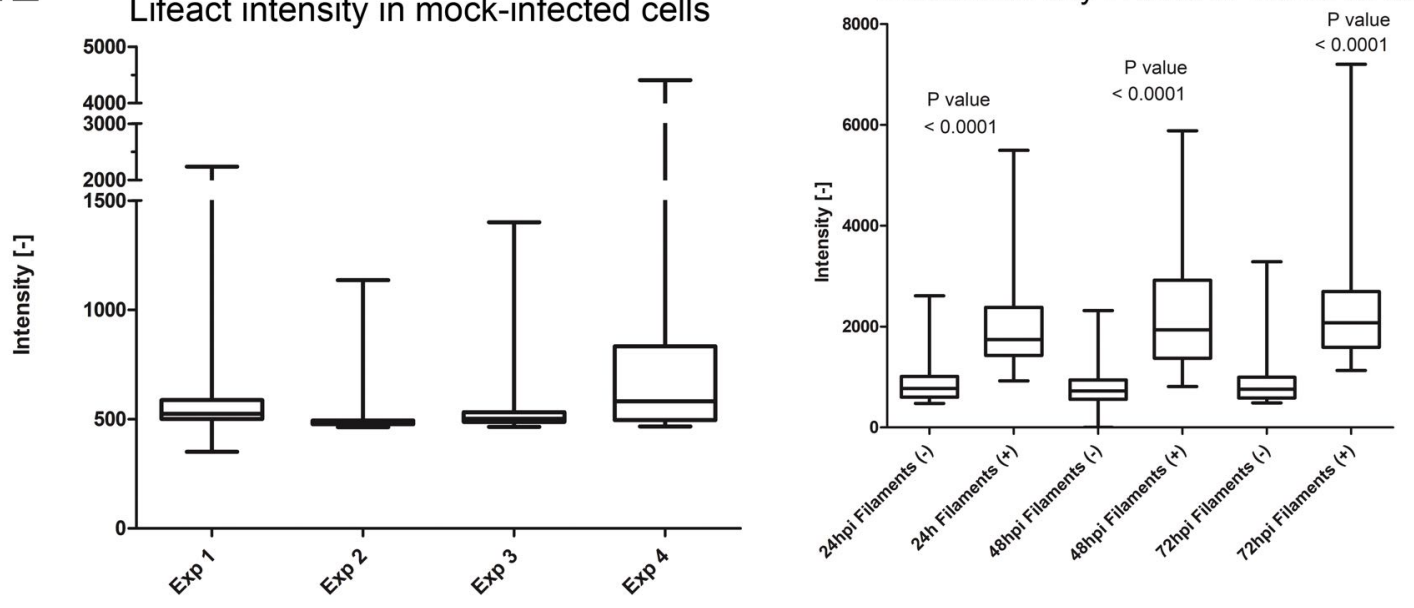
bioRxiv preprint doi: https://doi org/10.1101/641266; this version posted May 24 2019. The copyright holder for this preprint (which was not certified by peer review) is the author/funder, who has granted bioRxiv a license to display the preprint in perpetuity. It is made available under aCC-BY-NC-ND 4.0 International license.

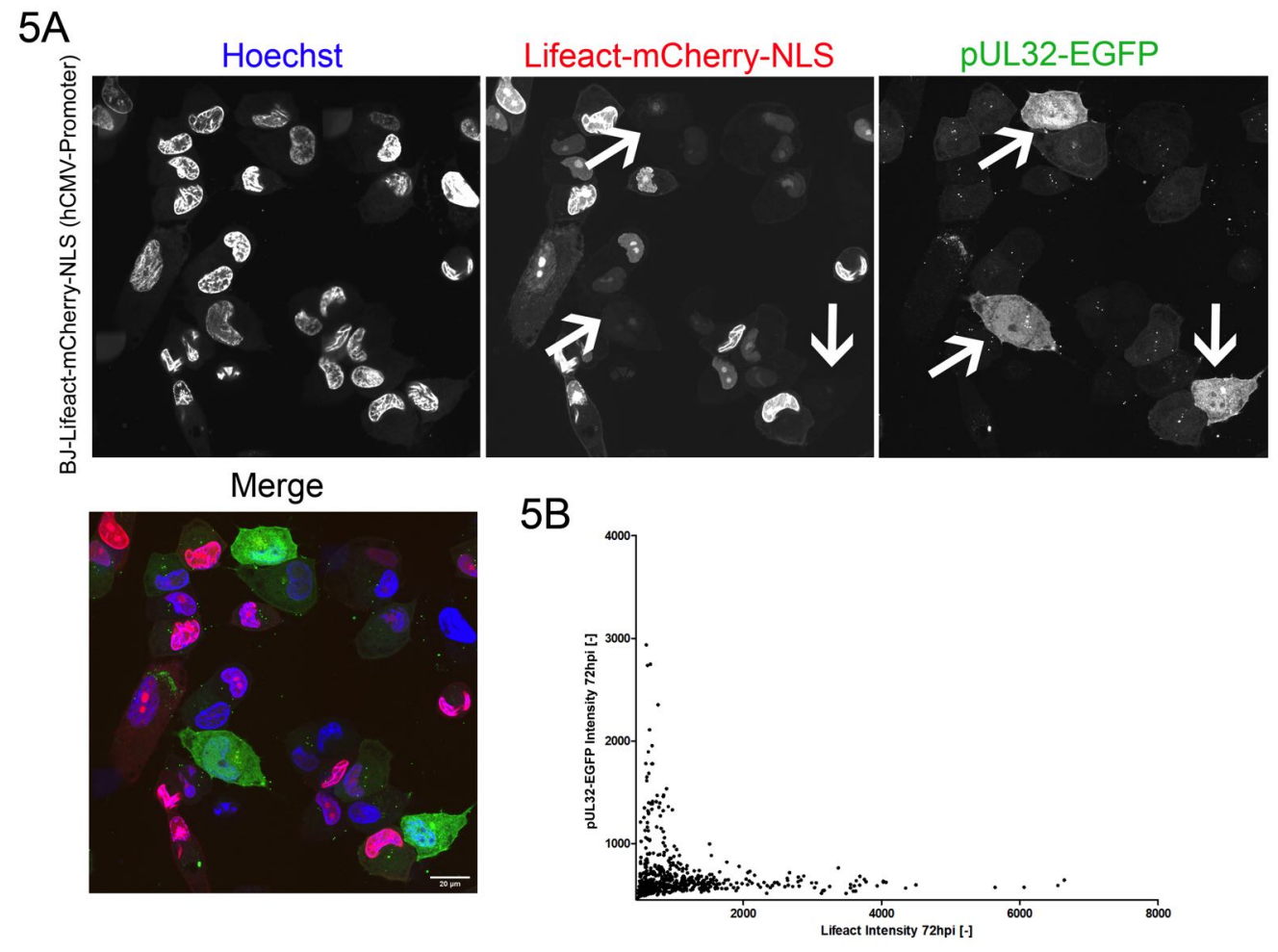


bioRxiv preprint doi: https://doi org/10.1101/641266; this version posted May 24, 2019. The copyright holder for this preprint (which was not certified by peer review) is the author/funder, who has granted bioRxiv a license to display the preprint in perpetuity. It is made available under aCC-BY-NC-ND 4.0 International license.

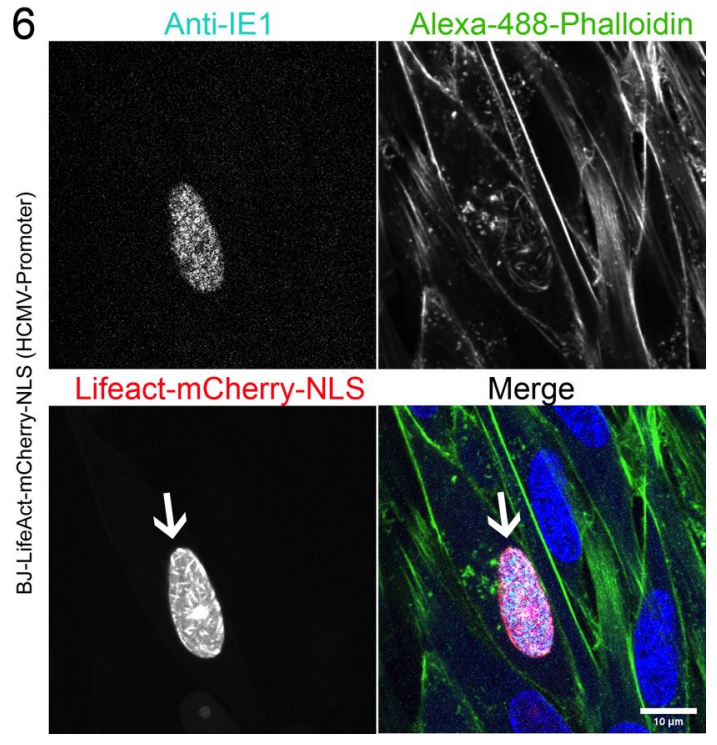


bioRxiv preprint doi: https://doi.org/10.1101/641266; this version posted May 24, 2019. The copyright holder for this preprint (which was

not certified by peer review) is the author/funder, who has granted bioRxiv a license to display the preprint in perpetuity. It is made available under aCC-BY-NC-ND 4.0 International license.

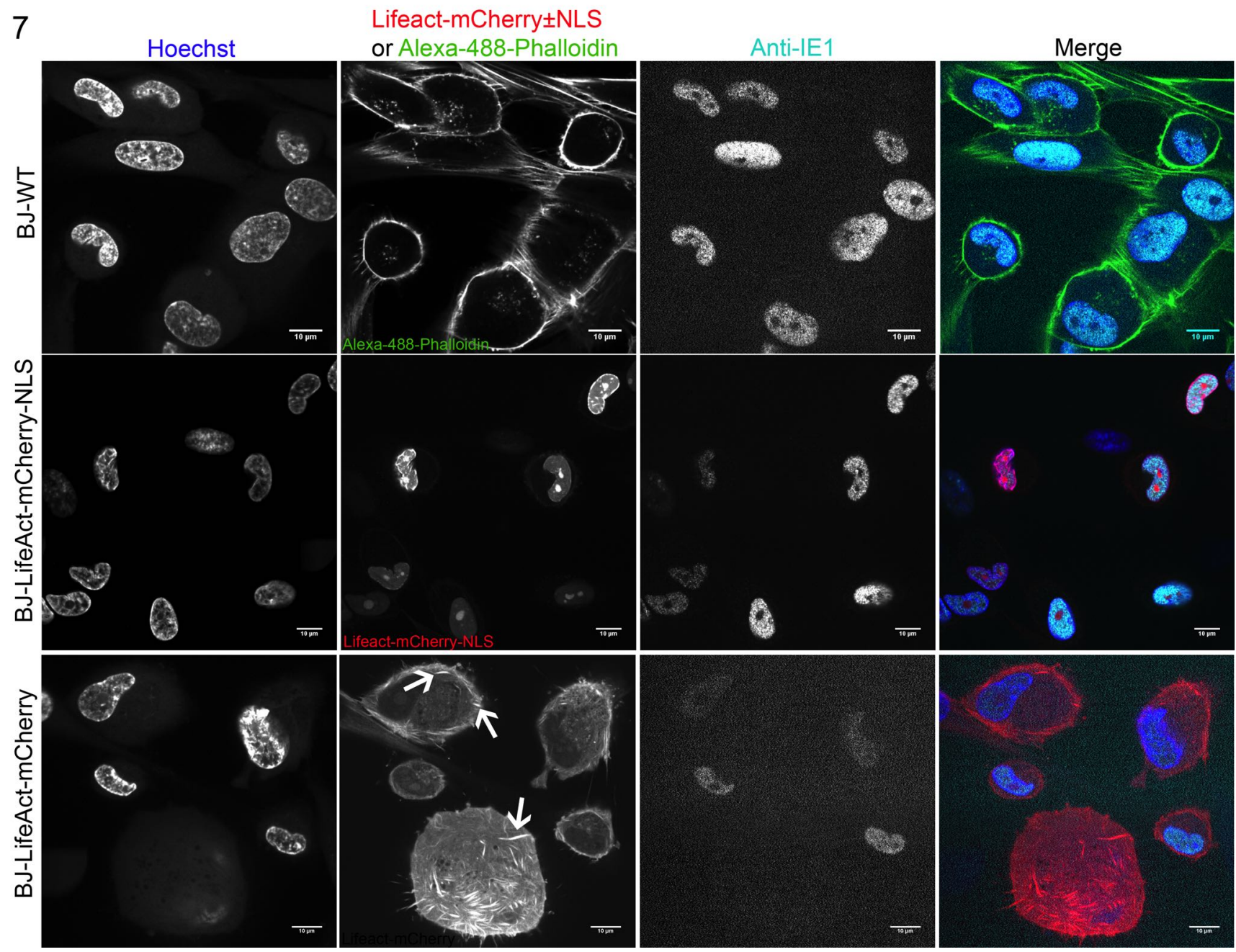


bioRxiv preprint doi: https://doi.org/10.1101/641266; this version posted May 24, 2019. The copyright holder for this preprint (which was

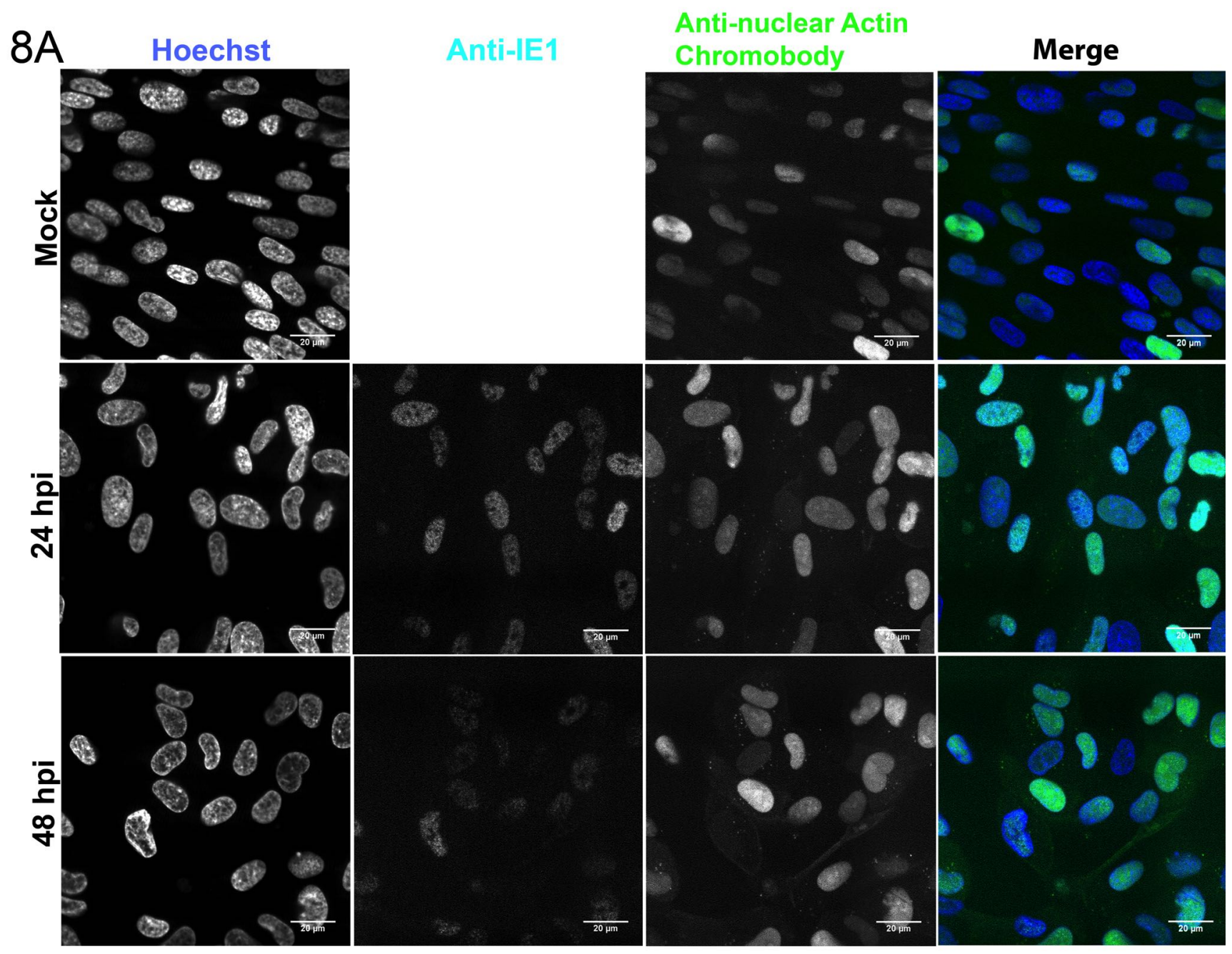

$8 \mathrm{~B}$

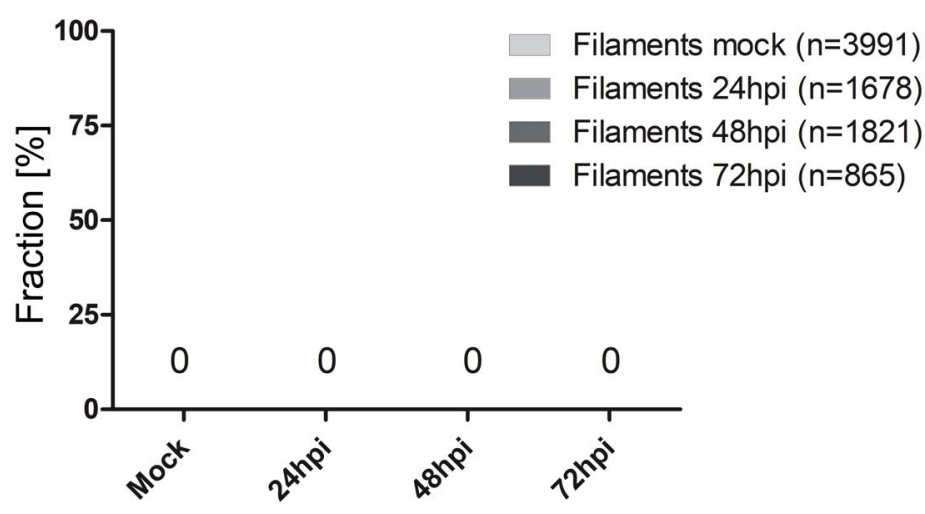


bioRxiv preprint doi: https://doi org/10.1101/641266; this version posted May 24 2019. The copyright holder for this preprint (which was not certified by peer review) is the author/funder, who has granted bioRxiv a license to display the preprint in perpetuity. It is made available under aCC-BY-NC-ND 4.0 International license.

9

Lifeact-mCherry-NLS

Merge
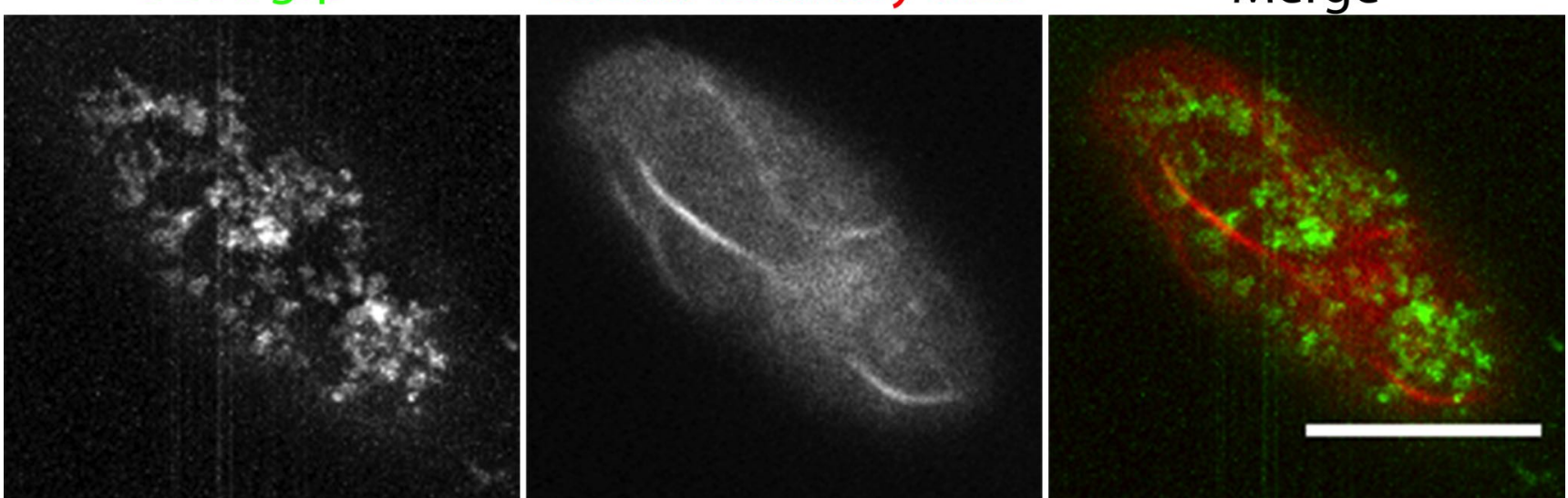
bioRxiv preprint doi: https://doi.org/10.1101/641266; this version posted May 24, 2019. The copyright holder for this preprint (which was not certified by peer review) is the author/funder, who has granted bioRxiv a license to display the preprint in perpetuity. It is made available under aCC-BY-NC-ND 4.0 International license.

$10 \mathrm{~A}$

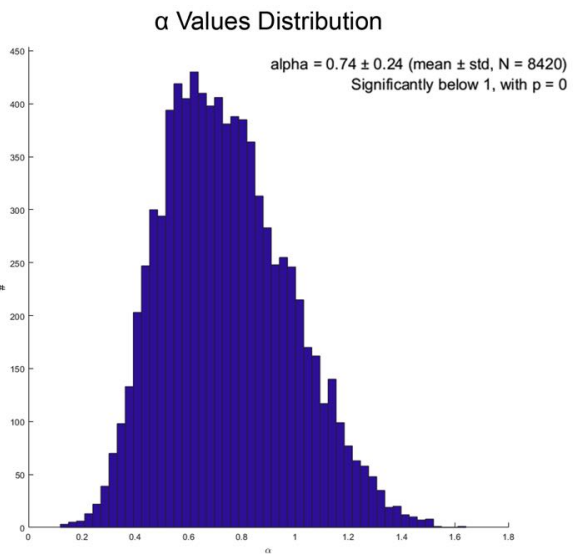

$10 \mathrm{~B}$

MSD-Plot

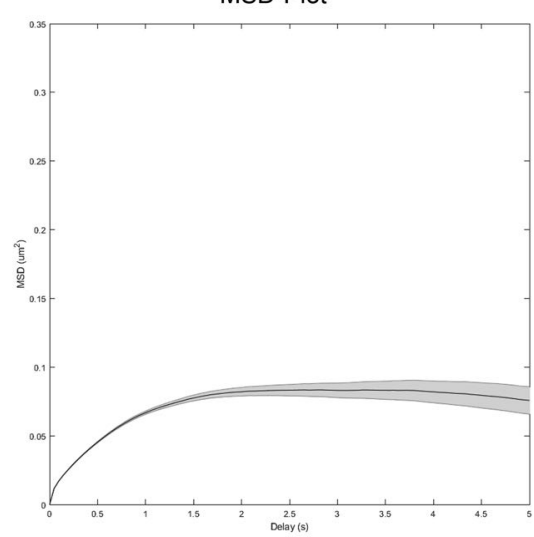


bioRxiv preprint doi: https://doi org/101101/641266; this version posted May 24 2019. The copyright holder for this preprint (which was not certified by peer review) is the author/funder, who has granted bioRxiv a license to display the preprint in perpetuity. It is made available under aCC-BY-NC-ND 4.0 International license.

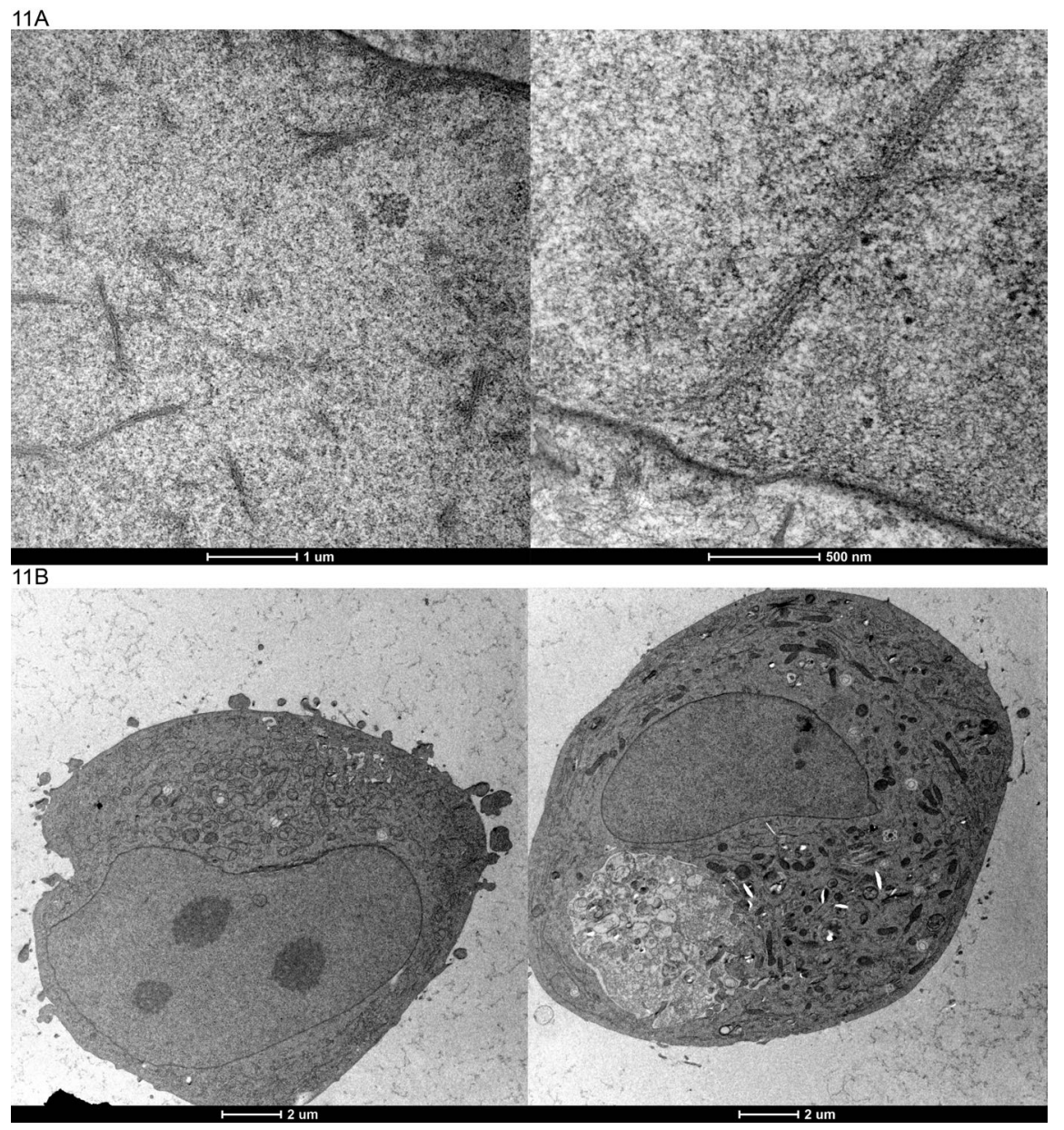

